\title{
Treatment of the extracranial carotid artery in tandem lesions during endovascular treatment of acute ischemic stroke: a systematic review and meta-analysis
}

\author{
Joyce Hellegering ${ }^{1}$, Maarten Uyttenboogaart ${ }^{2,3}$, Reinoud P. H. Bokkers ${ }^{3}$, Mostafa El Moumni ${ }^{1}$, \\ Clark J. Zeebregts ${ }^{1}$, Maarten J. van der Laan ${ }^{1}$ \\ ${ }^{1}$ Division of Vascular Surgery, Department of Surgery, ${ }^{2}$ Department of Neurology, ${ }^{3}$ Department of Radiology, Medical Imaging Center, University \\ Medical Center Groningen, University of Groningen, Groningen, The Netherlands \\ Contributions: (I) Conception and design: All authors; (II) Administrative support: None; (III) Provision of study materials or patients: None; (IV) \\ Collection and assembly of data: J Hellegering, MJ van der Laan, RPH Bokkers; (V) Data analysis and interpretation: All authors; (VI) Manuscript \\ writing: All authors; (VII) Final approval of manuscript: All authors. \\ Correspondence to: Maarten J. van der Laan, MSc, MD, PhD. Department of Vascular Surgery, University Medical Center Groningen, Hanzeplein 1, \\ 9713 GZ Groningen, The Netherlands. Email: M.J.vd.laan@umcg.nl.
}

\begin{abstract}
Endovascular treatment (EVT) is the standard treatment for patients with an acute ischemic stroke due to occlusion of large vessel occlusion (LVO). In $20 \%$ of patients, concomitant extracranial internal carotid artery (EICA) lesion is present. These tandem lesions (TL) offer a technical challenge. The treatment strategy for the treatment of the ipsilateral EICA is unclear. The aim of this review is to compare two treatment strategies for TL during EVT: balloon angioplasty (BA) only and immediate carotid artery stenting (iCAS). A systematic review and meta-analysis was performed. Data for each included study was extracted. For comparative studies a meta-analysis was performed. Functional outcome was expressed with the modified Rankin scale and safety endpoints were mortality and symptomatic intracranial hemorrhage (sICH). A total of 72 full text articles evaluating treatment of TL during EVT were screened. Sixteen iCAS and five comparative studies were included for meta-analysis. $53 \%$ of patients undergoing iCAS during EVT had good functional outcome in comparison to $45 \%$ of patients who underwent only BA. Mortality was comparable at $16 \%$ for both groups. The incidences of sICH were $8 \%$ and $4 \%$ for iCAS and BA respectively. In the meta-analysis, iCAS was associated with good functional outcome, with no significant differences in mortality and sICH with compared to BA. This study shows that treatment with iCAS of a simultaneously ipsilateral EICA lesion during EVT is associated with a favorable functional outcome compared to BA only with no significant difference in mortality or sICH. No conclusion could be drawn about the intracranial or extracranial first approach due to scarce of data. More studies are needed to determine long-term neurological outcomes, the necessity of re-interventions and optimal technical approach (intracranial or extracranial first).
\end{abstract}

Keywords: Balloon angioplasty (BA); carotid stenosis; stents; stroke; thrombectomy

Submitted Feb 29, 2020. Accepted for publication May 10, 2020.

doi: $10.21037 /$ atm-2020-cass-17

View this article at: http://dx.doi.org/10.21037/atm-2020-cass-17 


\section{Introduction}

In $20-30 \%$ of patients with acute ischemic stroke (AIS) caused by an intracranial large vessel occlusion (LVO) there is a concomitant high-grade stenosis or occlusion of the ipsilateral extracranial internal carotid artery (EICA). For decades, these tandem lesions (TL) had a poor prognosis due to a poor response on intravenous thrombolysis (IVT) alone, with a favorable outcome of only $30 \%$ and mortality rates up to $50 \%(1,2)$.

Recently, endovascular therapy (EVT), where the clot is mechanically extracted through endovascular access, has been proven effective and safe in patients with a LVO of the anterior circulation. EVT results in a higher recanalization rate and better functional outcomes than IVT alone and has been rapidly implemented in to national stroke guidelines and standard clinical care (3-5). For patients with a concomitant EICA lesion, EVT has also been shown to contribute to good functional outcome $(6,7)$. In those patients with a significant EICA stenosis, a balloon angioplasty (BA) is performed in order gain access to the intracerebral vessels for thrombectomy. The BA does however not lead to a definitive treatment of the lesion as there is often a recoil or residual-stenosis of the EICA (8).

Current literature does not provide a clear answer on whether the EICA lesion should also be stented in the acute setting. Guidelines recommend carotid endarterectomy (CEA) within 2 weeks after the first event in order to prevent a second event, however this is based on trials comparing CEA and stenting in a different population of patients with a subacute TIA or non-disabling stroke $(5,9-12)$. Acute patients may potentially benefit from early treatment as it further reduces the risk of recurrent stroke and the potential complication risks may be lower as endovascular access has already been obtained and the lesion has yet been passed with guidewires. The safety of immediate carotid artery stenting (iCAS) in the EVT group has not been fully elucidated. Since dual antiplatelet therapy (DAPT) is required after stenting, it may potentially lead to an increase in hemorrhagic complications in these patients with an acute stroke. Furthermore, it is unclear whether the EICA should be stented before or after recanalization of the occluded cerebral artery (13-17).

The aim of this review is to compare two treatment strategies, immediate CAS and BA, for tandem lesions during EVT and assess good functional outcome, efficacy and safety of external carotid artery treatment during EVT.

\section{Methods}

This systematic review was conducted in accordance to the Preferred Reporting Items for Systematic Review and MetaAnalysis (PRISMA) statement and Cochrane guideline of systematic reviews of interventions $(18,19)$.

\section{Data sources}

Databases of PubMed, Embase and Cochrane were searched till June $1^{\text {st }} 2019$ for eligible studies. Medical subject headings, terms and additional free entry terms for patient groups (patients with a tandem lesion, high-grade stenosis or occlusion, during acute ischemic stroke) and the treatment (BA, carotid artery stent) were used. The search strategy was formulated with the aid of a clinical librarian (Supplement file). The references of the selected papers were reviewed for the completion of the list of articles eligible for full text assessment.

\section{Study selection}

Two investigators (JH and ML) individually reviewed eligible titles and abstracts. Conflicts in inclusion were resolved through consensus. In case of disagreement a consensus was reached by consultation of a third reviewer (RB). Prespecified inclusion and exclusion criteria in our research protocol were used to select potentially eligible studies for full text analysis. Inclusion of a study followed if the study described the type of treatment of the EICA in TL during MT or delayed treatment after MT with separately reported outcomes. Prospective and retrospective observational studies, case series and randomized controlled trials were eligible for inclusion. Studies describing less than 10 patients were excluded from this review. The year of publication was no restriction on inclusion. Only full-text articles in English were included.

\section{Data extraction and quality assessment}

One investigator $(\mathrm{JH})$ extracted necessary information from the eligible articles. The data extracted was checked by sampling (RB, ML). Discrepancies were resolved by discussion between the investigators. If any of the main variables were missing or not reported separately, they were reported as missing data.

Data on the year of publication and year of last inclusion, 
number of included patients and type of procedure were extracted. Patient, procedural and outcome characteristics were recorded for all included articles.

All studies were non-randomized trials. Therefore, the methodological quality of the included studies was evaluated by the 'Methodological Index for Non-Randomized Studies' (MINORS) (20). Quality was categorized as: very low (0 to 6), low (7 to 10), fair (10 to 14) and good (>14).

\section{Outcome measures}

The primary goal of this review and meta-analysis was to estimate the incidence of good functional outcome at 90 days by means of modified Rankin Scale (mRS) score $\leq 2$ (21).

Secondary outcomes for this review were mortality at 90 days, defined as a mRS score of 6 or otherwise noted in the articles 90 days after intervention, symptomatic intracerebral hemorrhage (sICH) defined as any extravascular blood in the cranium or brain associated with neurological deterioration by means of NIHSS of $\geq 4$ points (22), successful reperfusion, defined as TICI $2 \mathrm{~b}$ or higher (23), (instent) re-stenosis or occlusion and cerebral hyperperfusion syndrome (CHS). We registered the preferable approach for recanalization (intracranial treatment versus extracranial treatment first respectively). One study defined sICH as any $\mathrm{PH}$ on following imaging (24) and one study only reported in-hospital mortality (25).

In addition, odds ratio's (ORs) were calculated to evaluate the association between type of intervention and the outcomes of favorable neurological outcome, sICH and mortality.

\section{Statistical analysis}

A logit transformation was used to calculate an overall estimate from studies reporting single proportions. After back transformation, pooled incidences with their $95 \%$ confidence intervals ( $95 \% \mathrm{CIs}$ ) were reported and illustrated in forest plots. Odds ratios with their $95 \%$ CIs were calculated and displayed for the comparative studies. All analyses were carried out using a random effects model.

To quantify heterogeneity, the Q-statistic, the betweenstudy variance $\left[\mathrm{T}^{2}\right.$, and standard deviation $\left.(\mathrm{T})\right]$ and the ratio of true heterogeneity to total observed variation $\left(\mathrm{I}^{2}\right)$ were computed. The between-study variance was estimated using the restricted maximum-likelihood estimator (REML). An $\mathrm{I}^{2}$ of $>50 \%$ represented a large degree of inconsistency across the studies.
In addition to funnel plots, both the Egger's test and Begg's rank test were used to detect publication bias when the number of included studies was at least 20 (26). A P values of $<0.05$ were considered statistically significant. All statistical analyses were performed using R 3.6.1 software using the meta package $(27,28)$.

\section{Results}

The search resulted in 391 records of which 319 were duplicates or irrelevant to the topic. After evaluation of the titles and abstracts, 72 articles were selected for fulltext assessment. Twenty-one studies met our inclusion criteria for this literature review $(7,14-17,24,25,29-42)$. Most frequent reason for exclusion $(\mathrm{n}=37)$ of an article was that data was not presented separately on outcome for the different treatment options or insufficiently reported. The review process is summarized in detail in Figure 1.

Fourteen of the 21 studies were retrospective cohort studies, six were comparative and one study was a prospective non-randomized observational study. The quality assessment of all 21 included studies is shown in Table 1. The methodological quality of the papers was low to fair. The MINORS score averaged 9.9 (9 to 11 , SD 0.7) out of 16 for non-comparative studies and 16.8 (15 to 18 , SD 1.1) out of 24 for comparative studies.

\section{Study characteristics}

The included studies enrolled a total of 1,758 patients (mean sample size, 84; range, 10-482). Of all included patients, 1,476 received iCAS (84\%) during EVT and 149 underwent BA $(8.4 \%)$. An extracranial first approach was favorable in 15 out of the 21 included studies. In total $7.6 \%$ was not treated during EVT. Because of the unclear method of how they were treated during EVT, we performed no further analysis within this subgroup.

\section{Immediate carotid artery stent placement for extracranial lesions during EVT}

Twenty-one of the 21 studies reported neurological outcome and safety for iCAS in patients undergoing EVT. Sixteen studies described only iCAS during EVT, of which only one study prospectively collected data. Table 1 shows the study characteristics in detail.

The pooled incidences are depicted in Figure 2. Good neurological outcome was seen in 53\% (95\% CI, 46-59\%). 


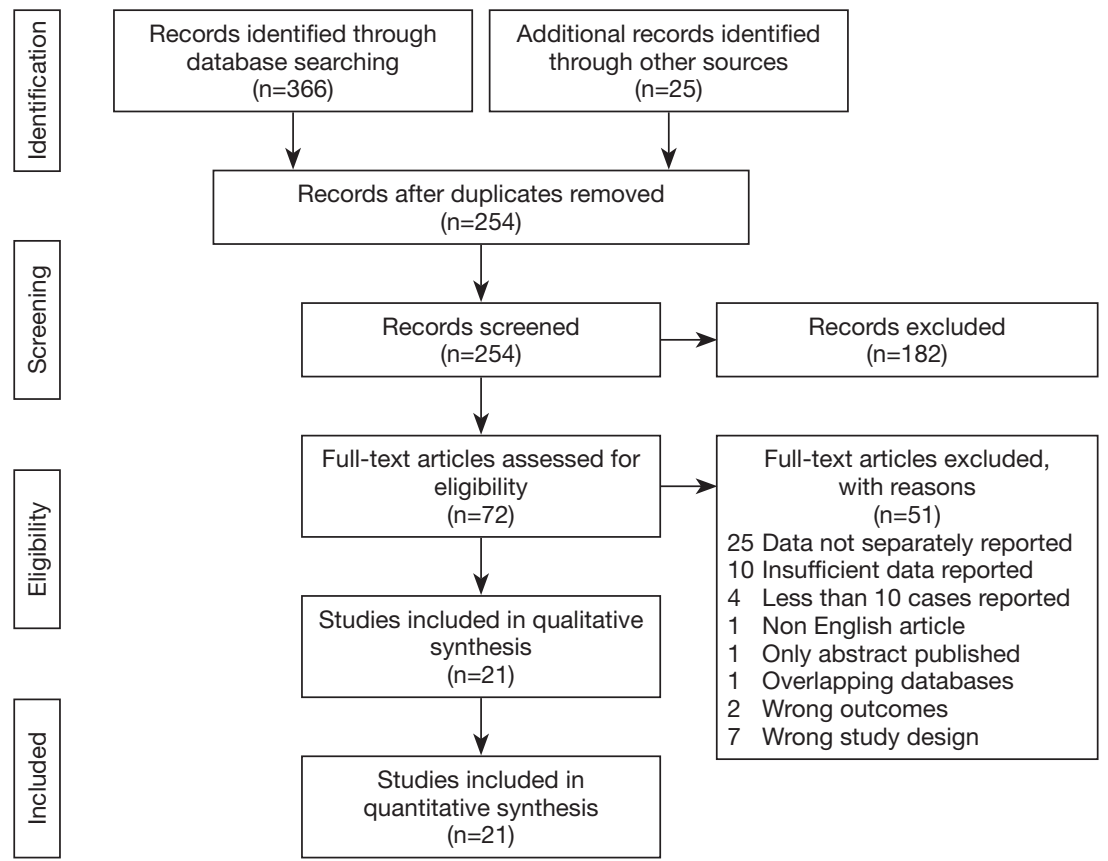

Figure 1 PRISMA flow diagram showing screening and selection of studies for systematic review (18).

The proportion of good neurological outcome varied largely across the studies $(\mathrm{Q}=116.52$, d.f. $=20, \mathrm{P}<0.0001$; $\left.\mathrm{T}^{2}=0.29 ; \mathrm{I}^{2}=82.8 \%\right)$. Mortality at 90 days was $16 \%(95 \%$ CI, 13-19\%). Heterogeneity was low in these studies (Q $=35.29$, d.f. $\left.=20, \mathrm{P}=0.02 ; \mathrm{T}^{2}=0.092 ; \mathrm{I}^{2}=43.3 \%\right)$. Mortality was mostly related to $\operatorname{sICH}(17,29,38,40)$ and pneumonia or sepsis $(30,40)$. Age, duration of treatment and time to recanalization were independently associated for all-cause mortality (7). In addition, prior IVT and the severity of the stroke was also correlated to all-cause mortality (16).

The incidence of sICH was $8 \%$ (95\% CI, 6-10\%). No substantial heterogeneity was seen in the studies $(\mathrm{Q}=30.01$, d.f. $\left.=20, P=0.07 ; T^{2}=0.15 ; I^{2}=33.4 \%\right)$. Successful cerebral reperfusion was reported in 19 studies and achieved in $79 \%$ (95\% CI, 74-84\%). There was a significant heterogeneity in this subgroup $\left(\mathrm{Q}=51.88\right.$, d.f. $=18, \mathrm{P}<0.0001 ; \mathrm{T}^{2}=0.25$; $\left.\mathrm{I}^{2}=65.3 \%\right)$. In-stent occlusion or stenosis on follow up imaging was documented in ten studies with an absolute incidence of $7.6 \%(n=39)$. In total, 25 asymptomatic in-stent restenosis or occlusions, two symptomatic instent occlusions after 24-48 hours and 12 acute in-stent occlusions were reported $(17,24,29,31,32,34,35,40-42)$. Only two studies reported on CHS, with an incidence of $2.4 \%(n=1)$ and $4.5 \%(n=1)(35,37)$.

Visual inspection of the funnel plots shown in Figure 3 indicate no presence of publication bias for favorable outcome, mortality and sICH. Using both the Egger's test and Begg's rank test, no statistically significant presence of publication bias was found (all $\mathrm{P}$ values $>0.4$ ). Due to missing data of two studies for successful reperfusion, there were not enough studies to perform a funnel plot for this outcome.

\section{$B A$ for extracranial lesions during $E V T$}

Five of the 21 studies reported neurological outcome and safety for iCAS in patients undergoing EVT. All studies were retrospectively comparative studies for iCAS (14-16,31,41). In all studies, the choice for BA was made by the discretion of the treating physicians, taking the access for distal treatment of the intracranial lesion into consideration.

Figure 4 shows the pooled incidence of endpoints in the BA group. Favorable outcome was seen in 46\% (95\% CI, 36-55\%). No substantial heterogeneity was seen in the studies $\left(\mathrm{Q}=4.89\right.$, d.f. $\left.=4, \mathrm{P}=0.30 ; \mathrm{T}^{2}=0.04 ; \mathrm{I}^{2}=18.2 \%\right)$. Overall mortality at 90 days was $14.7 \%$. Mortality at 90 days was equal to the iCAS group with $16 \%$ (95\% CI, $10-23 \%)$. No significant heterogeneity was found (Q $=3.71$, d.f. $\left.=4, \mathrm{P}=0.45 ; \mathrm{T}^{2}<0.0001 ; \mathrm{I}^{2}=0.0 \%\right)$. Three cases of sICH, 4\% (95\% CI, 1-9\%), were reported. No substantial heterogeneity was seen in the studies $(\mathrm{Q}=2.35$, d.f. $=4$, 


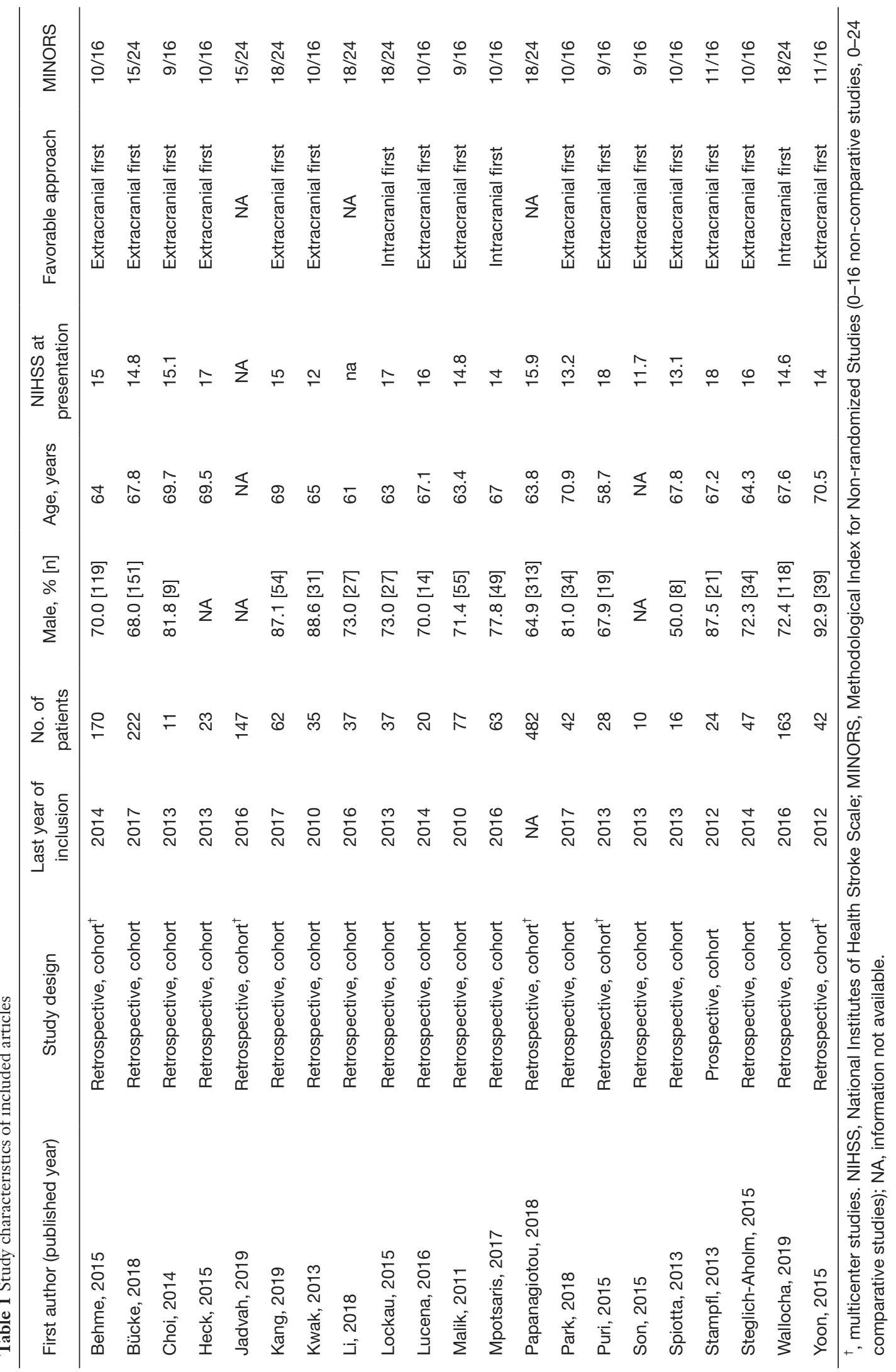




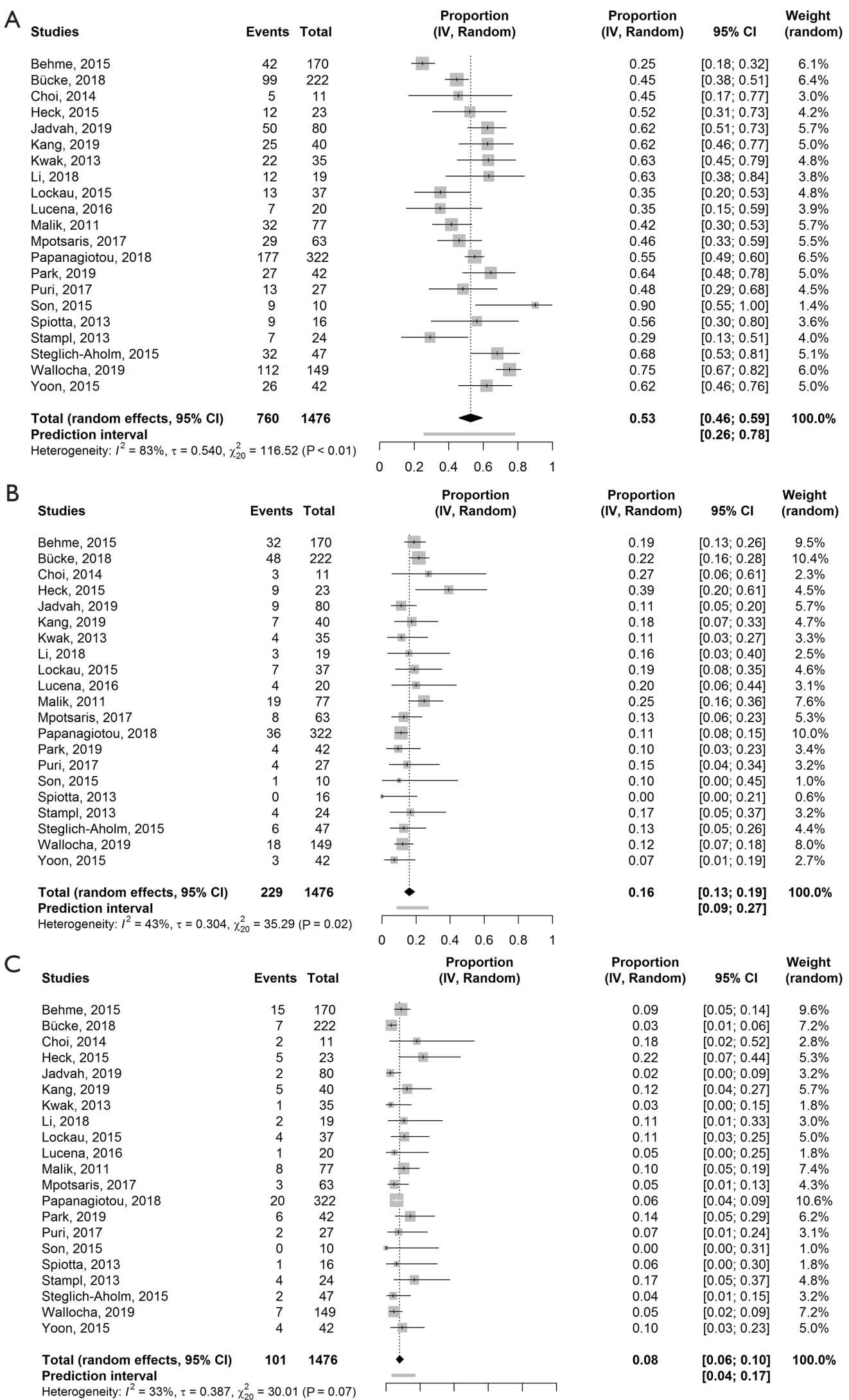




\begin{tabular}{|c|c|c|}
\hline Studies & Events & Total \\
\hline Behme, 2015 & 130 & 170 \\
\hline Bücke, 2018 & 197 & 222 \\
\hline Choi, 2014 & 4 & 11 \\
\hline Heck, 2015 & 17 & 23 \\
\hline Jadvah, 2019 & & 80 \\
\hline Kang, 2019 & 32 & 40 \\
\hline Kwak, 2013 & 26 & 35 \\
\hline $\mathrm{Li}, 2018$ & 14 & 19 \\
\hline Lockau, 2015 & 27 & 37 \\
\hline Lucena, 2016 & 18 & 20 \\
\hline Malik, 2011 & 58 & 77 \\
\hline Mpotsaris, 2017 & 55 & 63 \\
\hline Papanagiotou, 2018 & & 322 \\
\hline Park, 2019 & 33 & 42 \\
\hline Puri, 2017 & 19 & 27 \\
\hline Son, 2015 & 7 & 10 \\
\hline Spiotta, 2013 & 16 & 16 \\
\hline Stampl, 2013 & 15 & 24 \\
\hline Steglich-Aholm, 2015 & 42 & 47 \\
\hline Wallocha, 2019 & 137 & 149 \\
\hline Yoon, 2015 & 32 & 42 \\
\hline $\begin{array}{l}\text { Total (random effects, } 95 \% \mathrm{Cl} \text { ) } \\
\text { Prediction interval } \\
\text { Heterogeneity: } I^{2}=65 \%, \tau=0.495,\end{array}$ & 879 & 1476 \\
\hline
\end{tabular}

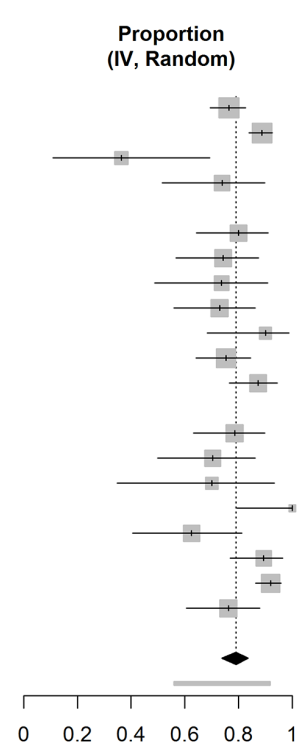

$\begin{array}{ccc}\begin{array}{c}\text { Proportion } \\ \text { (IV, Random) }\end{array} & \mathbf{9 5 \%} \mathbf{C l} & \begin{array}{c}\text { Weight } \\ \text { (random) }\end{array} \\ 0.76 & {[0.69 ; 0.83]} & 8.1 \% \\ 0.89 & {[0.84 ; 0.93]} & 7.8 \% \\ 0.36 & {[0.11 ; 0.69]} & 3.5 \% \\ 0.74 & {[0.52 ; 0.90]} & 4.8 \% \\ & 0.0 \% \\ 0.80 & {[0.64 ; 0.91]} & 5.6 \% \\ 0.74 & {[0.57 ; 0.88]} & 5.7 \% \\ 0.74 & {[0.49 ; 0.91]} & 4.4 \% \\ 0.73 & {[0.56 ; 0.86]} & 5.9 \% \\ 0.90 & {[0.68 ; 0.99]} & 2.8 \% \\ 0.75 & {[0.64 ; 0.84]} & 7.2 \% \\ 0.87 & {[0.77 ; 0.94]} & 5.8 \% \\ & & 0.0 \% \\ 0.79 & {[0.63 ; 0.90]} & 5.9 \% \\ 0.70 & {[0.50 ; 0.86]} & 5.3 \% \\ 0.70 & {[0.35 ; 0.93]} & 3.1 \% \\ 1.00 & {[0.79 ; 1.00]} & 1.0 \% \\ 0.62 & {[0.41 ; 0.81]} & 5.3 \% \\ 0.89 & {[0.77 ; 0.96]} & 4.8 \% \\ 0.92 & {[0.86 ; 0.96]} & 6.7 \% \\ 0.76 & {[0.61 ; 0.88]} & 6.0 \% \\ 0.79 & {[\mathbf{0 . 7 4 ; 0 . 8 4 ]}} & \mathbf{1 0 0 . 0 \%} \\ & {[\mathbf{0 . 5 6 ; 0 . 9 2 ]}} & \end{array}$

Figure 2 Forest plots showing the overall pooled incidence for immediate carotid stenting. (A) Favorable outcome (mRS 0-2 at 90 days); (B) 90 days mortality; (C) symptomatic intracranial hemorrhage; (D) successful reperfusion (TICI $\geq 2 \mathrm{~b}$ ).
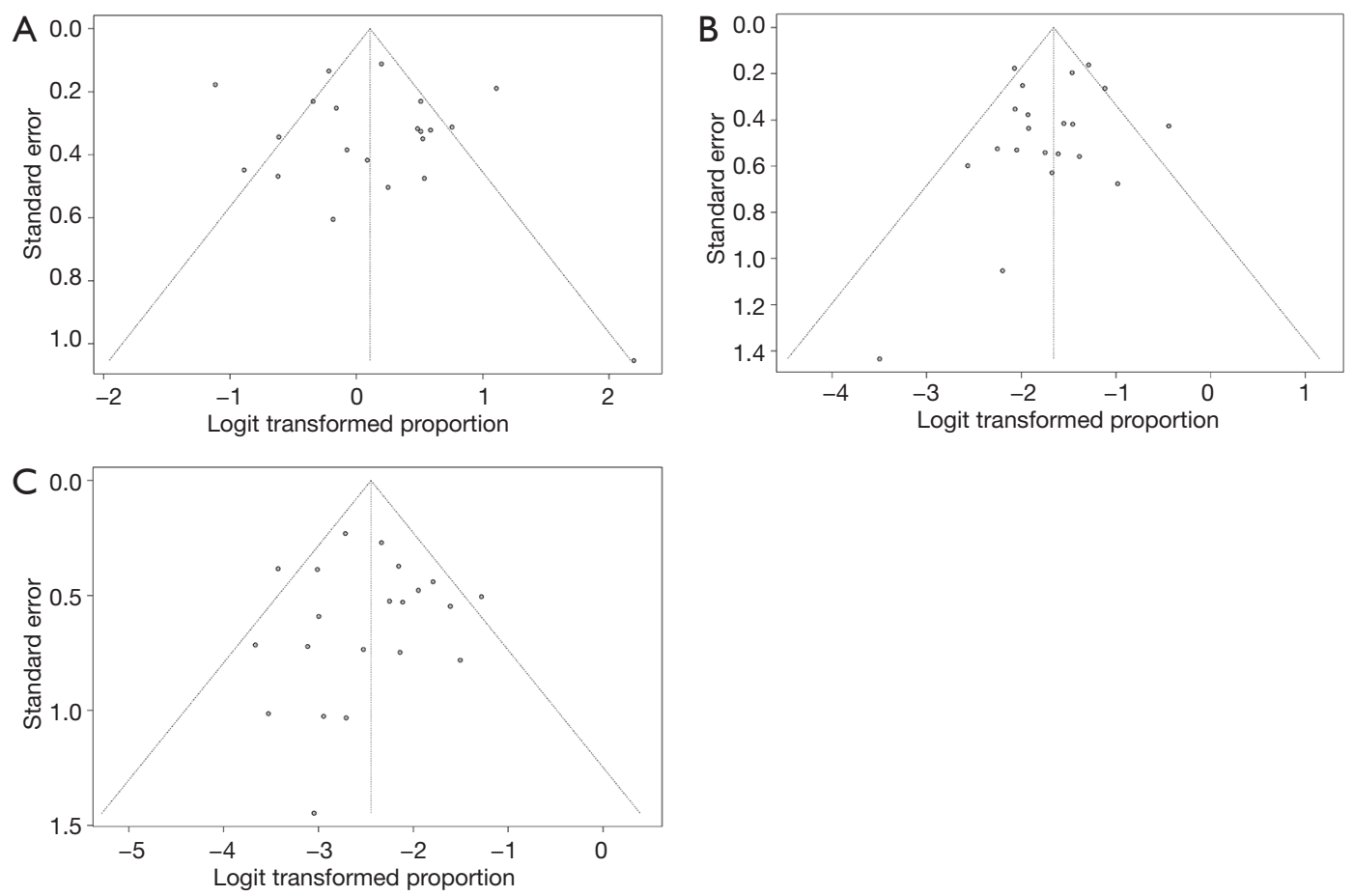

Figure 3 Funnel plots for publication bias on studies reporting immediate carotid stenting. (A) Favorable outcome (mRS); (B) mortality; (C) symptomatic intracranial hemorrhage. 


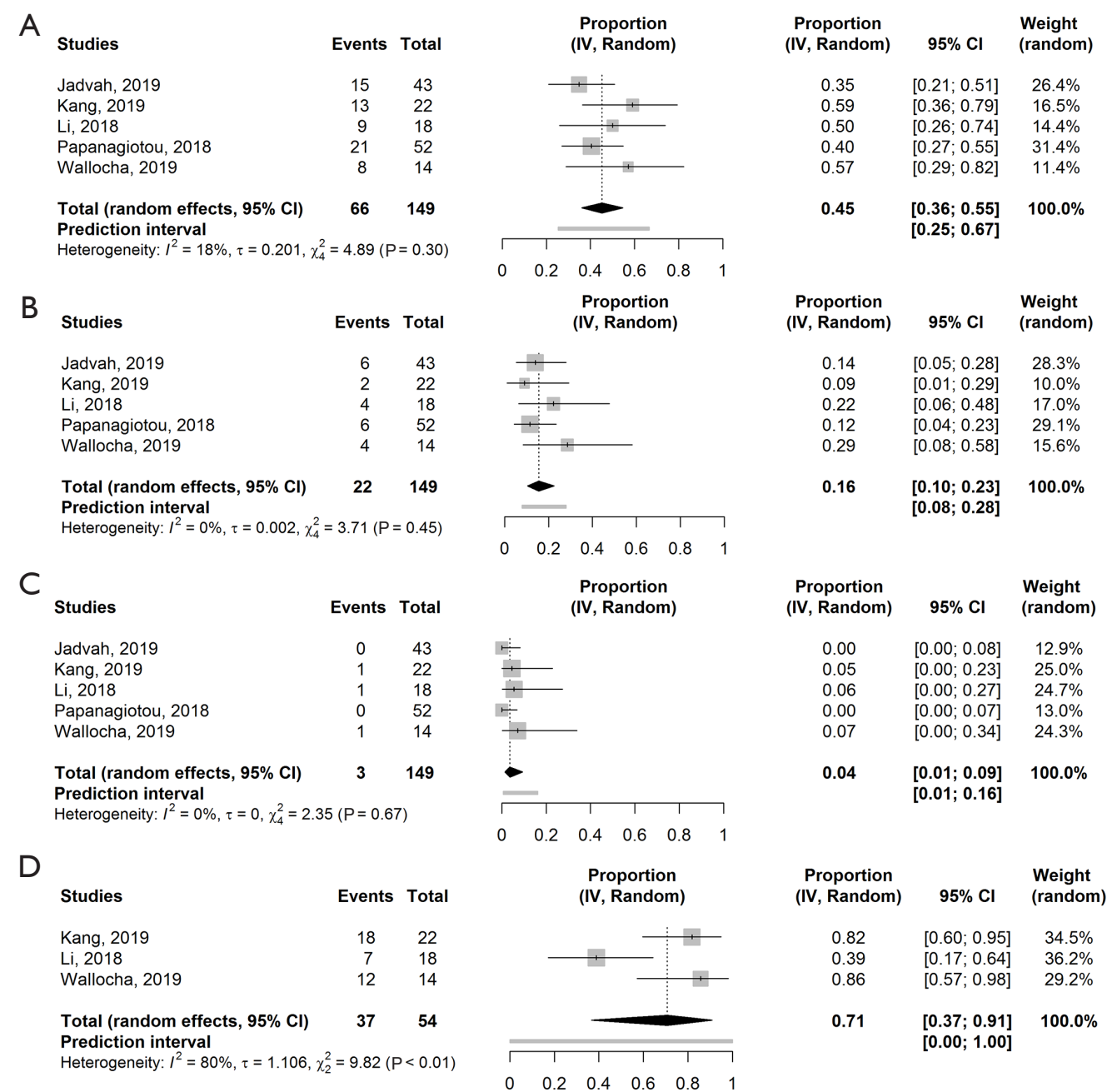

Figure 4 Forest plots showing the overall pooled incidence for BA. (A) Favorable outcome (mRS 0-2 at 90 days); (B) 90 days mortality; (C) symptomatic intracranial hemorrhage; (D) successful reperfusion (TICI $\geq 2 \mathrm{~b}$ ).

$\left.\mathrm{P}=0.67 ; \mathrm{T}^{2}=0.0 ; \mathrm{I}^{2}=0.0 \%\right)$. Reperfusion was only reported in four articles, with a successful reperfusion rate of $71 \%$ (95\% CI, 37-91\%) $(15,31,36,41)$. Heterogeneity was low in these studies $\left(\mathrm{Q}=9.82\right.$, d.f. $\left.=2, \mathrm{P}=0.01, \mathrm{~T}^{2}=1.22 ; \mathrm{I}^{2}=79.6 \%\right)$. Asymptomatic post procedural EICA re-occlusion was described in $8 \%(\mathrm{n}=8)$ on ultrasound $(15,31,41)$. Nor acute nor symptomatic occlusions were reported. Of the two studies who reported CHS, no CHS occurred in the BA group.

\section{iCAS versus $B A$ alone during $E V T$}

Five studies compared the iCAS to BA alone. All studies were nonrandomized observational studies. In the five included comparative studies for iCAS or BA, iCAS during EVT was associated with better functional outcome at 90 days than BA only (OR 1.99, 95\% CI: 1.35-2.93\%). Heterogeneity was low in these studies $\left(\mathrm{Q}=2.5\right.$, d.f. $=4, \mathrm{P}=0.64 ; \mathrm{T}^{2}=0.00$; $\left.\mathrm{I}^{2}=0.0 \%\right)$. No association was found between iCAS and BA related to 90-day mortality (OR $1.27,95 \%$ CI: $0.73-2.19$ ) and sICH (OR 0.48, 95\% CI: 0.16-1.45) (Figure 5A,B,C). There was no significant heterogeneity in these subgroups ( $\mathrm{Q}=3.26$, d.f. $=4, \mathrm{P}=0.52 ; \mathrm{T}^{2}=0.0 ; \mathrm{I}^{2}=0.0 \%$ and $\mathrm{Q}=2.00$, d.f. $=4, \mathrm{P}=0.74 ; \mathrm{T}^{2}=0.0 ; \mathrm{I}^{2}=0.0 \%$ for mortality and $\mathrm{sICH}$ respectively). These findings follow the trend of the separately performed pooled incidence analysis. 


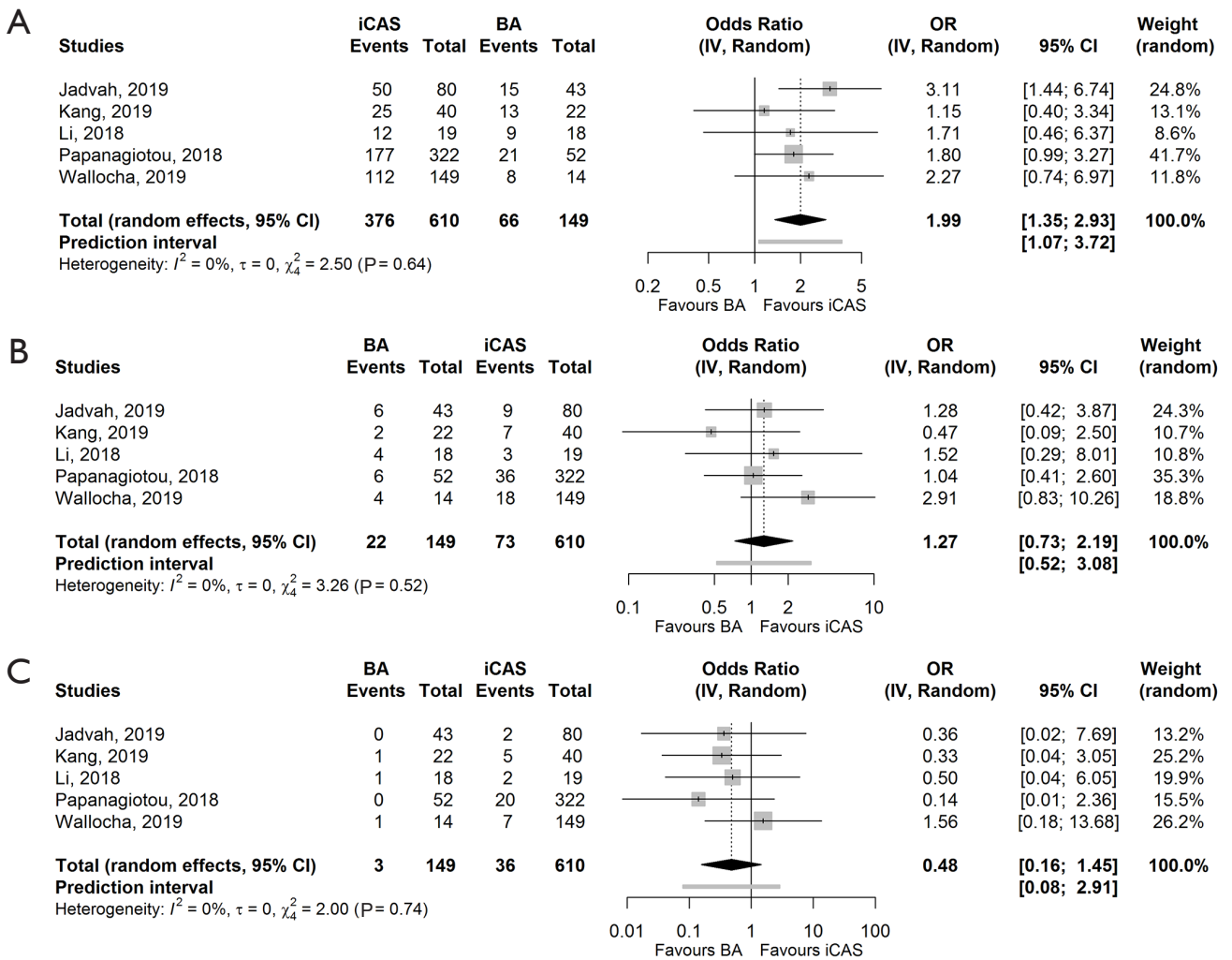

Figure 5 Meta-analysis for iCAS vs. BA. (A) Favorable outcome (mRS 0-2 at 90 days); (B) 90 days mortality; (C) symptomatic intracranial hemorrhage.

\section{Extracranial treatment versus intracranial treatment first}

In most studies approach considerations of the TL was reported, favoring extracranial approach first. Successful reperfusion in this group was seen in $84.7 \%$ with a 90 -day good functional outcome of $47.2 \%$. sICH was reported in $4.5 \%$ and 90 -day mortality of $18.1 \%(7,17,24,30,32$ $34,37,38,42)$.

In case of intracranial treatment first, successful reperfusion was seen in $80.3 \%$ and 90 -day good functional outcome of $51.8 \%$ with $17.8 \%$ mortality rate and an incidence of $12.5 \%$ for $\operatorname{sICH}(15,32)$.

Only three studies reported outcomes of intracranial treatment first of which two discuss their motive for intracranial treatment first in more detail. Lockau et al. (32) found that the mean times from first puncture of the groin till reperfusion of intracranial occlusion and overall perfusion, including the ipsilateral EICA, were significantly shorter when the intracranial thrombectomy was performed prior to iCAS (43.1 \pm 30.8 vs. $110.8 \pm 43.0 \mathrm{~min}, \mathrm{P}<0.001,58.6 \pm$
26.1 vs. $130.2 \pm 45.1 \mathrm{~min}, \mathrm{P}<0.01$ intracranial treatment vs. extracranial treatment first respectively).

Another study (34) also compared both approaches. However, there was no significant difference in time from groin puncture till reperfusion [110 minutes (range, 15-208 minutes) vs. 125 minutes (range, 45-212 minutes), $\mathrm{P}=0.42$, intracranial treatment $v$ s. extracranial treatment first respectively]. The authors also referred to an incidental finding with a potential benefit for extracranial approach first of spontaneous distal, e.g., intracranial, thrombus dissolution in $24 \%(n=4 / 17)$, without seeing this observation in the intracranial approach first.

\section{Discussion}

This systematic review and meta-analysis evaluate the safety and efficacy of treatment of EICA lesion in combination with an intracranial lesion during EVT by means of iCAS or BA. The current evaluation shows that despite the fact of having a tandem lesion, iCAS during EVT was associated 
with a good 90-day functional outcome, without significant differences in mortality or bleeding complications in comparison to BA alone.

In 2016, the HERMES collective published the first meta-analysis on EVT for LVO. This study was focused on the intracranial LVO and confirmed the efficacy of EVT in this subgroup with a favorable functional outcome in $46.0 \%$ (3). The current review shows a comparable or better functional outcome at 90 days for EICA lesions treated with iCAS with similar incidence of mortality, with a slightly higher incidence of sICH.

Bleeding complications like sICH due to DAPT are feared after treatment with stents in the acute phase, mainly because of the associated high morbidity and mortality afterwards. A few of the included studies showed a high incidence of sICH. A possible explanation for these high rates is the use of a strict and alternate high dosed antiplatelet regime. In up to $31 \%$ of a treated subgroup with Glycoprotein IIb/IIIA inhibitors (Abciximab, Eptifibatide respectively), sICH was reported $(29,38)$. Treatment protocols of both studies were changed after these findings. Unfortunately, literature is ambiguous about the impact and effects of DAPT during CAS, nor is there consensus on a standardized antiplatelet regime for (immediate) CAS. As an example, none of the included studies in this review have applied the same antiplatelet protocol with a high heterogeneity among the studies. A meta-analysis in 2016 evaluated the evidence for DAPT after elective endovascular arterial procedures, with primary endpoint restenosis and stent thrombosis, with secondary endpoint bleeding complications (43). Most studies involved coronary literature, two studies involved CAS. They found no significant evidence for superiority for DAPT over monotherapy for neither restenosis nor for stent thrombosis. The two included carotid studies $(44,45)$ both stopped prematurely because of the large differences in outcome between monotherapy and DAPT, in favor of DAPT with less neurological complications (stroke, transient ischemic attacks or intracerebral bleedings). However, none of latter studies looked at functional outcome nor mortality.

Despite most studies did not report CHS, hyperperfusion after CAS is an infamous complication. Huibers et al. (46) investigated in their meta-analysis if CHS in clinically relevant in relation to procedural stroke rate following elective CAS. A pooled estimate for CHS of $3.5 \%$ was found, not associated with the type of anesthesia. Approximately $8 \%$ of CHS occurred after hospital discharge. However, the authors expect an underreporting due to a majority of the studies only report in hospital outcomes.

Little is known about the long-term outcomes for TL treated with EVT. Ten included studies reported on acute stent occlusions and in-stent stenosis during follow up. Only one study documented the need for delayed treatment in both groups (15). In 22 cases of BA, delayed carotid revascularization was required by form of delayed iCAS $(n=1)$ or carotid endarterectomy (CEA) $(n=7)$. No additional treatment after iCAS were reported in this cohort.

Long-term results of the EVA-3S, CREST and ICSS suggest a benefit-risk balance in favor of CEA over CAS on short term results after treatment. This difference is mainly driven by a lower risk of AIS during the intervention. Both techniques were associated with similar long-term risks of recurrent mainly non-disabling stroke, myocardial infarction and death up to 10 years after intervention (10-12). In case of EVT in the presence of a tandem lesion, a larger chance on disrupting the plaque and creating micro-emboli stroke due to passing of the carotid lesion is inherent to the executed EVT. Earlier drawn conclusions may therefore not be applicable for patient with TL undergoing EVT. However, insufficient data is available to make any statements on long-term results in this specific population.

Several included studies described predictors for good functional outcome. A TICI score of 3 and early neurological improvement are factors for a favorable outcome at 90 days (35). Other predictors of good functional outcome months are age $(7,15,16,34,41)$, low NIHSS score at baseline $(14,16)$ and procedure time to recanalization $(7,15,41)$. An additional hour between arrival at the emergency department and puncture of the femoral artery was associated with a $22 \%$ reduction of TICI $2 \mathrm{~b} / 3$ reperfusion (47). In addition, in procedures ranging from 20 to $60 \mathrm{~min}$ the functional outcome was good in $79 \%$ of patients (41). In procedures lasting more than $60 \mathrm{~min}$, only $50 \%$ of the patients had a mRS $0-2$ at long-term followup. According to the adagium "time is brain", a fast passage of the EICA and intracranial recanalization may further improve functional outcomes. It is possible to conclude that an intracranial first approach may be beneficial (48).

This review holds some inherent limitations. Firstly, the quality of this systematic review and meta-analysis is directly related to the quality of the predominantly uncontrolled studies included. All but one study were retrospective series and therefore subjected to a marked 
risk of selection bias. Most studies did not meet an adequate score on the MINORS tool. None of the studies prospectively randomized their study size nor was there mention of an unbiased assessment of the study endpoints. Secondly, there is a considerable heterogeneity among the studies due to different treatment plans, different primary outcomes and different antiplatelet regimes. As already discussed earlier on, the difference in antiplatelet regime may affect the neurological outcomes and complication rates. Thirdly, most studies depicted poorly the type of lesions found during EVT in the ipsilateral EICA. These different presentations may require different approaches with possible effect on outcome. Fourthly, only ten studies noted the presence of potential in-stent stenosis and reocclusions of the EICA after treatment. It is not sufficiently clear what the implications are on 90-day good functional outcome nor mortality nor the long-term outcome with BA of iCAS. Lastly, hemorrhagic complications were reported variously in the included studies. Several studies also reported parenchymal hemorrhages (subdivided or as one), asymptomatic ICH or even by any hemorrhage. Due to the lacking synchronicity of definitions, it is possible that an underestimation of relevant bleeding complications is represented in this review.

\section{Conclusions}

In conclusion, this systematic review shows that treatment with iCAS of a simultaneously ipsilateral EICA lesion during EVT is associated with a favorable functional outcome at 90 days compared to BA only with no significant increase in mortality or sICH. No conclusion could be drawn about the intracranial or extracranial first approach due to scarce of data. More studies are needed to determine long-term neurological outcomes, the necessity of reinterventions and optimal technical approach (intracranial or extracranial first). Prospective randomized control trials are mandatory, as selection or publication bias, may have influenced the results.

\section{Acknowledgments}

Funding: None.

\section{Footnote}

Provenance and Peer Review: This article was commissioned by the Guest Editor (Dr. Kosmas I. Paraskevas) for the series "Carotid Artery Stenosis and Stroke: Prevention and Treatment Part I" published in Annals of Translational Medicine. The article was sent for external peer review organized by the Guest Editor and the editorial office.

Conflicts of Interest: All authors have completed the ICMJE uniform disclosure form (available at http://dx.doi. org/10.21037/atm-2020-cass-17). The series "Carotid Artery Stenosis and Stroke: Prevention and Treatment Part I" was commissioned by the editorial office without any funding or sponsorship. The authors have no other conflicts of interest to declare.

Ethical Statement: The authors are accountable for all aspects of the work in ensuring that questions related to the accuracy or integrity of any part of the work are appropriately investigated and resolved.

Open Access Statement: This is an Open Access article distributed in accordance with the Creative Commons Attribution-NonCommercial-NoDerivs 4.0 International License (CC BY-NC-ND 4.0), which permits the noncommercial replication and distribution of the article with the strict proviso that no changes or edits are made and the original work is properly cited (including links to both the formal publication through the relevant DOI and the license). See: https://creativecommons.org/licenses/by-nc-nd/4.0/.

\section{References}

1. Kim YS, Garami Z, Mikilik R, et al. Early Recanalization Rates and Clinical Outcomes in Patients With Tandem Internal Carotid Artery / Middle Cerebral Artery Occlusion and Isolated Middle Cerebral Artery Occlusion. Stroke 2005;36:869-71.

2. Rubiera M, Ribo M, Degado-Mederos R, et al. Tandem Internal Carotid Artery/Middle Cerebral Artery Occlusion. An independent predictor of poor outcome after systemic thrombolysis. Stroke 2006;37:2301-5.

3. Goyal M, Menon BK, van Zwam WH, et al. Endovascular thrombectomy after large-vessel ischaemic stroke: a metaanalysis of individual patient data from five randomised trials. Lancet 2016;387:1723-31.

4. Berkhemer OA, Fransen PS, Beumer D, et al. A Randomized Trial of Intraarterial Treatment for Acute Ischemic Stroke. N Engl J Med 2015;372:11-20.

5. Powers WJ, Rabinstein AA, Ackerson T, et al, 2018 Guidelines for the early management of patients with acute 
Page 12 of 13

ischemic stroke: a guideline for healthcare professionals from the American Heart Association/American Stroke Association. Stroke 2018;49:e46-110.

6. Gory B, Haussen DC, Piotin M, et al. Impact of intravenous thrombolysis and emergent carotid stenting on reperfusion and clinical outcomes in patients with acute stroke with tandem lesion treated with thrombectomy: a collaborative pooled analysis. Eur J Neurol 2018;25:1115-20.

7. Bücke P, Perez MA, AlMatter M, et al. Functional outcome and safety of intracranial thrombectomy after emergent extracranial stenting in acute ischemic stroke due to tandem occlusions. Front Neurol 2018;9:940.

8. Maus, V, Borggrefe, J, Behme, D. et al. Order of treatment matters in ischemic stroke: mechanical thrombectomy first, then carotid artery stenting for tandem lesions of the anterior circulation. Cerebrovasc Dis 2018;46:59-65.

9. Rothwell PM, Eliasziw M, Gutnikov SA, et al. Endarterectomy for symptomatic carotid stenosis in relation to clinical subgroups and timing of surgery. Lancet 2004;363:915-24.

10. Brott TG, Howard H, Gary PH, et al. Long-term results of stenting versus endoarterectomy for carotid-artery stenosis. N Engl J Med 2016;374:1021-31.

11. Bonati LH, Dobson J, Featerstone RL, et al. Longterm outcomes after stenting versus endarterectomy for treatment of symptomatic carotid stenosis: the International Carotid Stenting Study (ICSS) randomised trial. Lancet 2015;385:529-38.

12. Mas JL, Arquizan C, Calvet D, et al. Long-Term FollowUp Study of Endarterectomy Versus angioplasty in Patients With Symptomatic Severe Carotid stenosis Trial. Stroke 2014;45:2750-6.

13. Akpinar CK, Gürkaş E, Aytac E. Carotid angioplastyassisted mechanical thrombectomy without urgent stenting may be a better option in acute tandem occlusions. Interv Neuroradiol 2017;23:405-11.

14. Jadvah AP, Zaidat OO, Liebeskind DS, et al. Emergent management of tandem lesions in acute ischemic stroke, analysis of the STRATIS registry. Stroke 2018;50:428-33.

15. Kang DH, Kim YW, Hwang YH, et al. Endovascular recanalization of acute tandem cervical carotid and intracranial occlusions: efficacy of cervical balloon angioplasty alone then intracranial target recanalization strategy. World Neurosurg 2019;126:e1268-75.

16. Papanagiotou P, Haussen D, Turjman F, et al. Carotid Stenting With Antithrombotic Agents and Intracranial Thrombectomy Leads to the Highest Recanalization Rate
Hellegering et al. Treatment of carotid artery lesions during EVT

in Patients With Acute Stroke With Tandem Lesions. JACC Cardiovasc Interv 2018;11:1290-9.

17. Choi JY, Lee JI, Lee TJ, et al. Emergent Recanalization with Stenting for Acute Stroke due to Athero-Thrombotic Occlusion of the Cervical Internal Carotid Artery : A Single Center Experience. J Korean Neurosurg Soc 2014;55:313-20.

18. Moher D, Liberati A, Tetzlaff J, et al. Preferred Reporting Items for Systematic Reviews and Meta-Analyses: The PRISMA Statement PLoS Med 2009;6:e1000097.

19. Higgins JPT, Thomas J, Chandler J, et al. Cochrane Handbook for Systematic Reviews of Interventions version 6.0 (updated July 2019). Available online: www.training. cochrane.org/handbook

20. Slim K, Nini E, Forestier D, et al. Methodological index for non-randomized studies (MINORS): development and validation of a new instrument. ANZ J Surg 2003;73:712-6.

21. Rankin J. Cerebral vascular accidents in patients over the age of 60: II. Prognosis. Scot Med J 1957;2:200-15.

22. von Kummer R, Broderick JP, Campbell BCV, et al. The Heidelberg Bleeding Classification. Stroke 2015;46:2981-6.

23. Malik AM, Vora NA, Lin R, et al. Endovascular treatment of tandem extracranial/intracranial anterior circulation occlusions. Preliminary single-center experience. Stroke 2011;42:1653-7.

24. Behme D, Mpotsaris A, Zeyen P, et al. Emergency stenting of the extracranial internal carotid artery in combination with anterior circulation thrombectomy in acute ischemic stroke; a retrospective multicenter study. Am J Neuroradiol 2015;36:2340-5.

25. Fugate JE, Klunder AM, Kallmes DF, et al. What Is Meant by "TICI"? Am J Neuroradiol 2013;34:1792-7.

26. Furuya-Kanamori L, Xu C, Lin L, et al. P value-driven methods were underpowered to detect publication bias: analysis if Cochrane review meta-analyses. J Clin Epidemiol 2020;118:86-92.

27. Balduzzi S, Rucker G, Schwarzer G. How to preform a meta-analysis with R: a practical tutorial. EBMH 2019.

28. R Core Team. R: a Language and Environment for statistical computing. Vienna Austria. Available online: www.R-project.org

29. Heck DV, Brown MD. Carotid stenting and intracranial thrombectomy for treatment of acute stroke due to tandem occlusions with aggressive antiplatelet therapy may be associated with a high incidence of intracranial hemorrhage. J Neurointerv Surg 2015;7:170-5. 
30. Kwak HS, Hwang SB, Jin GY, et al. Predictors of functional outcome after emergency carotid artery stenting and intra-arterial thrombolysis for treatment of acute stroke associated with obstruction of the proximal internal carotid artery and tandem downstream occlusion. Am J Neuroradiol 2013;34:841-6.

31. Li W, Chen Z, Dai Z, et al. Management of acute tandem occlusions; stent-retriever thrombectomy with emergency stenting or angioplasty. J Int Med Res 2018;46:2578-86.

32. Lockau H, Liebig T, Henning T, et al. Mechanical thrombectomy in tandem occlusion: procedural considerations and clinical results. Neuroradiology 2015;57:589-98.

33. Lucena AF, Castro-Afonso LH, Monsignore LM, et al. Carotid artery stenting in the context of endovascular treatment of acute ischemic stroke. Arq Neuropsiquiatr 2016;74:212-8.

34. Mpotsaris A, Kabbasch C, Borggrefe J, et al. Stenting of the cervical internal carotid artery in acute stroke management: The Karolinska experience. Interv Neuroradiol 2017;23:159-65.

35. Park SE, Choi DS, Baek HJ, et al. Endovascular therapy of acute ischemic stroke related to tandem occlusion: comparison of occlusion and severe stenosis of the proximal cervical internal carotid artery. BR J Radiol 2019;92:20180051.

36. Puri AS, Kühn AL, Kwan HJ, et al. Endovascular treatment of tandem vascular occlusions in acute ischemic stroke. J Neurointerv Surg 2015;7:158-63.

37. Son S, Choi DS, Oh MK, et al. Emergency carotid artery stenting in patients with acute ischemic stroke due to occlusion or stenosis of the proximal internal carotid artery: a single-center experience. J Neurointerv Surg 2015;7:238-44.

38. Spiotta AM, Lena J, Vargas J, et al. Proximal to distal approach in the treatment of tandem occlusions causing an acute stroke. J Neurointerv Surg 2015;7:164-9.

Cite this article as: Hellegering J, Uyttenboogaart M, Bokkers RPH, El Moumni M, Zeebregts CJ, van der Laan MJ. Treatment of the extracranial carotid artery in tandem lesions during endovascular treatment of acute ischemic stroke: a systematic review and meta-analysis. Ann Transl Med 2020;8(19):1278. doi: 10.21037/atm-2020-cass-17
39. Stampfl S, Ringleb PA, Möhlenbruch M, et al. Emergency cervical internal carotid artery stenting in combination with intracranial thrombectomy in acute stroke. Am J Neuroradiol 2014;35:741-6.

40. Steglich-Arnholm H, Holtmannspötter M, Kondziella D, et al. Thrombectomy assisted by carotid stenting in acute ischemic stroke management: benefits and harms. J Neurol 2015;262:2668-75.

41. Wallocha M, Chapot R, Nordmeyer H, et al. Treatment methods and early neurologic improvement after endovascular treatment of tandem occlusions in acute ischemic stroke. Front Neurol 2019;10:127.

42. Yoon W, Kim BM, Kim DI, et al. Outcomes and prognostic factors after emergent carotid artery stenting for hyperacute stroke within 6 hours of symptoms onset. Neurosurgery 2015;76:321-9.

43. Peeters Weem SMO, van Haelst STW, den Ruijter HM, et al. Lack of Evidence for Dual Antiplatelet Therapy after Endovascular Arterial Procedures: A Meta-analysis. Eur J Vasc Endovasc Surg 2016;52:253-62.

44. Dalainas I, Nano G, Bianchi P, et al. Dual Antiplatelet Regime Versus Acetyl-acetic Acid for Carotid Artery Stenting. Cardiovasc Intervent Radiol 2006;29:519-21.

45. McKevitt FM, Randall MS, Cleveland TJ, et al. The Benefits of Combined Anti-platelet Treatment in Carotid Artery Stenting. Eur J Vasc Endovasc Surg 2005;29:522-7.

46. Huibers AE, Westerink J, de Vries EE, et al. Editor's Choice - Cerebral Hyperperfusion Syndrome After Carotid Artery Stenting: A Systematic Review and Metaanalysis. Eur J Vasc Endovasc surg 2018;56:322-33.

47. Bourcier R, Goyal M, Liebeskind DS, et al. Association of Time From Stroke Onset to groin Puncture With Quality of Reperfusion After Mechanical Thrombectomy. A Metaanalysis of Individual Patient Data From 7 Randomized Clinical Trials. JAMA Neurol 2019;76:405-11.

48. Gomez CR. Editorial: Time is brain! J Stroke Cerebrovasc Dis $1993 ; 3: 1-2$. 


\section{Search strategies}

\section{PubMed}

("Stroke"[Mesh] OR stroke[tiab] OR CVA[tiab] OR cerebrovascular accident*[tiab]) AND ("Thrombolytic Therapy"[Mesh] OR "Mechanical Thrombolysis"[Mesh] OR "Infusions, Intra-Arterial"[Mesh] OR "Endovascular Procedures"[Mesh:NoExp] OR intraarterial[tiab] OR intra-arterial[tiab] OR thromboly*[tiab]) AND ("Carotid Arteries"[Mesh] OR "Carotid Stenosis"[Mesh] OR caroti*[tiab] OR extracranial occlus*[tiab]) AND tandem*[tiab]

\section{Embase}

('cerebrovascular accident'/exp OR (stroke OR CVA OR 'cerebrovascular accident*'):ab,ti) AND ('fibrinolytic therapy'/exp OR 'mechanical thrombectomy'/exp OR 'intraarterial drug administration'/de OR 'intracarotid drug administration'/exp OR 'endovascular surgery'/de OR (intraarterial OR 'intra-arterial' OR thromboly* OR 'acute ischemic stroke' OR 'acute ischaemic stroke' OR (acute AND ('ischemic stroke' OR 'ischaemic stroke'))):ab,ti) AND ('carotid artery'/de OR 'carotid artery bifurcation'/exp OR 'common carotid artery'/exp OR 'external carotid artery'/ exp OR 'internal carotid artery'/exp OR 'carotid artery obstruction'/exp OR caroti*:ab,ti) NOT (('animal'/exp OR 'nonhuman'/exp) NOT 'human'/exp) NOT 'conference abstract'/it AND tandem

\section{Cochrane}

(stroke OR CVA OR cerebrovascular accident*) AND (intraarterial OR "intra-arterial" OR thromboly* OR thrombectom* OR (acute AND ("ischemic stroke" OR "ischaemic stroke")) OR "fibrinolytic therapy" OR intracaroti* OR endovascular*) AND (caroti*) AND tandem* 